\title{
One Digital Health Is FAIR
}

\author{
Arriel BENIS ${ }^{\mathrm{a}, 1}$ and Oscar TAMBURIS ${ }^{\mathrm{b}}$ \\ ${ }^{\text {a }}$ Faculty of Industrial Engineering and Technology Management, \\ Holon Institute of Technology, Holon, Israel \\ ${ }^{\mathrm{b}}$ Department of Veterinary Medicine and Animal Productions, \\ University "Federico II", Naples, Italy
}

\begin{abstract}
The One Digital Health framework aims at transforming future health ecosystems and guiding the implementation of a digital technologies-based systemic approach to caring for humans' and animals' health in a managed surrounding environment. To integrate and to use the data generated by the ODH data sources, "FAIRness" stands as a prerequisite for proper data management and stewardship.
\end{abstract}

Keywords. One Health, Digital Health, One Digital Health, FAIR, FAIRness

\section{Introduction}

The One Digital Health (ODH) [1] framework allows the analysis of the digital health ecosystem components through different perspectives focusing on how technologies may support healthcare and well-being activities. An ODH intervention can support the management of such a web (i.e., human, animal, and environmental) of digital interconnections. To integrate and to use the data generated by the ODH data sources, "FAIRness" stands as a prerequisite for proper data management and stewardship [2,3]: the FAIR Principles provide guidelines for the publication of those digital resources (or digitalities) whose combination and implementations set up the shape of an ODH intervention, for making them Findable, Accessible, Interoperable, and Reusable [2,3]. Besides the existing FAIRness metrics, new ones need therefore to be developed within new contexts such as ODH. How does the ODH framework support FAIR?

\section{Materials and Methods}

The ODH "Steering Wheel" is built around 2 keys (One Health, Digital Health), 3 perspectives (individual health and well-being, population and society, ecosystem), and 5 dimensions (citizens' engagement, education, environment, human and veterinary health care, Healthcare Industry 4.0) [1]. The digital technologies (digital-ities) to be singled out and analyzed within the ODH dimensions prism aim to: increase animal welfare and account for "how" humans affect animals' lives, health, and interactions; relate to how technology has been embedded in human experiences and activities; aim to support the management and governance of the complex interactions between humans,

1 Corresponding author, Dr. Arriel Benis, Faculty of Industrial Engineering and Technology Management, Holon Institute of Technology, Golomb St 52, Holon, 5810201, Israel; e-mail: arrielb@ hit.ac.il. 
animals, and their ecosystems. FAIR spotlights the capacity of computational systems to find, access, interoperate and reuse data with a minimum of human interventions, due to the increasing volume, velocity, and variability of data. This means that, to get to an optimal ODH-ness (i.e. an effective supply of an ODH intervention), each investigated digitality involved is globally evaluated, also in terms of its FAIRness with adapted metrics.

\section{Results}

The developing ODH-ness compliance analysis assessment comprises a FAIRness evaluation management component. The design and deployment of an ODH intervention imply for data to be: Findable because the digitalities involved are part of the study and collection of all the data related to the interconnection between systems' needs; Accessible via standardized protocols, to leverage the available common substrates of data, information, and knowledge stemming from digital biodiversity; Interoperable as a consequence of the awareness to establish an ecosystem capable of seamless, secure health data exchange and processing, to deal with the shared risks between animal and human populations; Reusable to allow a systematic, continuous, and intelligent integration of big, smart, and multidimensional data to be exchanged by the digitalities involved.

\section{Discussion and Conclusion}

Despite the efforts performed to deploy Open Science data stewardship, the comprehensive view that the $\mathrm{ODH}$ framework can provide is still lacking. ODH requires adopting new kinds of data environments, technologies, and standards. The COVID-19 pandemic has forced people to dwell on the close relationships among the environment, animals, and humans. Initiatives as the FAIR4Health project encouraged the health research community to FAIRify, share, and reuse their datasets derived from publiclyfunded research initiatives [4]. The ODH is FAIR. ODH pillars are the availability (findability, accessibility) of human, animal, and environmental data allowing a unified understanding of complex interactions (interoperability) over time (reusability). It is therefore a prolific landscape that joins FAIR for global health as an interdisciplinary and unifying layer by developing "fair" ODH interventions.

\section{References}

[1] Benis A, Tamburis O, Chronaki C, Moen A. One Digital Health: A Unified Framework for Future Health Ecosystems. J. Med. Internet Res. 2021; 23: e22189.

[2] Wilkinson MD, Dumontier M, Aalbersberg IJ, et al. The FAIR Guiding Principles for scientific data management and stewardship. Scientific Data. 2016; 3: 1-9.

[3] Wilkinson MD, Sansone S-A, Schultes E, et al. A design framework and exemplar metrics for FAIRness. Scientific Data. 2018; 5: 1-4.

[4] FAIR4Health, https://www.fair4health.eu/en/project\# (Accessed on September $9^{\text {th }}, 2021$ ). 